\title{
Temperature Effect on the Bandgaps of Surface and Bulk Acoustic Waves in Two-Dimensional Phononic Crystals
}

\author{
Zi-Gui Huang and Tsung-Tsong Wu
}

\begin{abstract}
In this paper, we analyzed the temperature effect on two-dimensional phononic crystals. Bandgap variations of both of the bulk modes and surface modes due to changing of temperature in an air/quartz band structure from 0 to $50^{\circ} \mathrm{C}$ were calculated and discussed. The results show that the elastic bandgaps can be enlarged or reduced by adjusting the temperature of the band structure. The temperature effects potentially can be used for fine-tuning of the phononic bandgap frequency.
\end{abstract}

\section{INTRODUCTION}

$\mathrm{R}$ ECENT successful application of photonic crystals has led to a rapidly growing interest in the analogous acoustic effects in periodic elastic structures, called phononic crystals. Research in bandgaps of bulk acoustic waves of phononic structures have been carried out in the past [1]-[8]. The dispersion relations and bandgap properties of the transverse polarization modes for periodic, elastic composites were analyzed and discussed [1]-[3]. The dispersion relations of mixed polarization modes and the experimental evidence for the existence of absolute acoustic bandgaps also were described [4], [5]. Bandgaps for outof-plane propagation of elastic waves were computed in the phononic band structure of the anisotropic infinite square array of parallel quartz rods embedded in an epoxy matrix [6], and the effects of the orientation of square rods on the acoustic bandgaps in a two-dimensional phononic crystal (solid/air) were discussed [7], [8]. In [9]-[12], the multiple scattering theory was applied to study the bandgaps of bulk waves in three-dimensional periodic acoustic composites and the band structure of a phononic crystal consisting of complex and frequency-dependent Lamé coefficients. The finite-difference, time-domain method was applied to interpret the experimental data of two-dimensional systems consisting of cylinders of fluids (mercury, air, and oil) inserted periodically in a finite slab of aluminum host [13].

Surface wave propagation on layered superlattices with traction-free surface parallel to the layers, has been explored extensively in the past [14]. However, investigations into surface wave properties of solids, in which the periodic modulation occurs on the traction-free surface did not take place until quite recently [15]-[20]. In [15] and [16], the cal-

Manuscript received June 8, 2004; accepted September 1, 2004.

The authors are with the Institute of Applied Mechanics, National Taiwan University, Taipei 106, Taiwan (e-mail: wutt@ndt.iam.ntu.edu.tw). culations for surface waves on a square and hexagonal superlattice, consisting of cubic (AlAs/GaAs) and isotropic ( $\mathrm{Al} /$ polymer) materials were reported, and $\mathrm{Wu}$ et al. [17] extended these works by studying the phononic bandgaps of the surface and bulk acoustic modes in two-dimensional phononic structures consisting of general anisotropic materials. Vines et al. [18], [19] conducted an experimental study on the surface waves generated by a line-focus acoustic lens at the water-loaded surfaces of a number of twodimensional superlattices that intersect the surface normally. The propagation of Scholte-like acoustic waves at the liquid-loaded surfaces of periodic structures also has been studied [20].

Although many theoretical and experimental studies have been done on two-dimensional periodic structures, the temperature effect for different polarization propagation modes have so far not been investigated. In this paper, we use the plane-wave expansion (PWE) method adopted in [17] to calculate the variations of bandgap widths of the quasishear vertical (SV), quasishear horizontal (SH), quasilongitudinal (L), and surface acoustic wave (SAW) modes due to the temperature changes. After a detailed examination of the dispersion curves with different temperature, we found that the behaviors of the variations of bandgap width are quite distinct and obvious. We shall refer to this obvious phenomenon as the fine-tuning of the bandgap in such a band structure.

\section{Acoustic Waves in Two-Dimensional PhONONIC CRYSTALS}

In an inhomogeneous, linear, elastic, anisotropic medium with no body force, the equation of motion for the displacement vector $\mathbf{u}(\mathbf{r}, t)$ can be written as:

$$
\rho(\mathbf{r}, T) \ddot{u}_{i}(\mathbf{r}, t)=\partial_{j}\left[C_{i j m n}(\mathbf{r}, T) \partial_{n} u_{m}(\mathbf{r}, t)\right],
$$

where $\mathbf{r}=(\mathbf{x}, z)=(x, y, z)$ is the position vector, $T$ is the temperature variable, $t$ is the time variable, $\rho(\mathbf{r}, T)$ and $C_{i j m n}(\mathbf{r}, t)$ are the position-dependent and temperaturedependent mass density and elastic stiffness tensor, respectively. In the following, we consider a phononic crystal composed of a two-dimensional periodic array ( $x-y$ plane) of material $A$, embedded in a background material $B$. Due to the spatial periodicity, the material constants, $\rho(\mathbf{x}, T)$ and $C_{i j m n}(\mathbf{x}, T)$ can be expanded at temperature $T$ and 
in Fourier series, with respect to the two-dimensional reciprocal lattice vectors (RLV), $\mathbf{G}=\left(G_{1}, G_{2}\right)$, as:

$$
\begin{aligned}
\rho(\mathbf{x}, T) & =\sum_{\mathbf{G}} e^{i \mathbf{G} \cdot \mathbf{x}} \rho_{\mathbf{G}}(T), \\
C_{i j m n}(\mathbf{x}, T) & =\sum_{\mathbf{G}} e^{i \mathbf{G} \cdot \mathbf{x}} C_{\mathbf{G}}^{i j m n}(T),
\end{aligned}
$$

where $\rho_{\mathbf{G}}(T)$ and $C_{\mathbf{G}}^{i j m n}(T)$ are the corresponding Fourier coefficients at temperature $T$.

To use the Bloch's theorem and to expand the displacement vector $\mathbf{u}(\mathbf{r}, t)$ in Fourier series for the analyses of the surface and bulk waves, we have:

$$
\mathbf{u}(\mathbf{r}, t)=\sum_{\mathbf{G}} e^{i \mathbf{k} \cdot \mathbf{x}-i \omega t}\left(e^{i \mathbf{G} \cdot \mathbf{x}} \mathbf{A}_{\mathbf{G}} e^{i k_{z} z}\right),
$$

where $\mathbf{k}=\left(k_{1}, k_{2}\right)$ is the Bloch wave vector, $\omega$ is the circular frequency, $k_{z}$ is the wave number along the $z$ direction, and $\mathbf{A}_{\mathbf{G}}$ is the amplitude of the displacement vector. We note that, as the component of the wave vector $k_{z}$ equals zero, (4) degenerates into the displacement vector of a bulk acoustic wave. On substituting (2), (3), and (4) into (1), and after collecting terms systematically, we obtain the generalized eigenvalue problem as:

$$
\left(\mathbf{A} k_{z}^{2}+\mathbf{B} k_{z}+\mathbf{C}\right) \cdot \mathbf{U}=0
$$

where $\mathbf{A}, \mathbf{B}$, and $\mathbf{C}$ are $3 n \times 3 n$ matrices, and they are functions of the Bloch wave vector $\mathbf{k}$, components of the two-dimensional RLV, circular frequency $\omega$, the Fourier coefficients of mass density $\rho_{\mathbf{G}}(T)$ and components of elastic stiffness tensor $C_{\mathbf{G}}^{i j m n}(T) . n$ is the total number of RLV used in the Fourier expansion, and $\mathbf{U}=\left[\begin{array}{lll}A_{\mathbf{G}^{\prime}}^{1} & A_{\mathbf{G}^{\prime}}^{2} & A_{\mathbf{G}^{\prime}}^{3}\end{array}\right]^{T}$ is the eigenvector. The expressions of the matrices $\mathbf{A}, \mathbf{B}$, and $\mathbf{C}$ are listed in [17]. Where $\mathbf{G}$ and $\mathbf{G}^{\prime}$ are the twodimensional reciprocal lattice vectors.

Eq. (5) can be solved by introducing $\mathbf{V}=k_{z} \mathbf{U}$ and rewritten in the form as [17]:

$$
\left[\begin{array}{cc}
\mathbf{0} & \mathbf{I} \\
-\mathbf{A}^{-1} \mathbf{C} & -\mathbf{A}^{-1} \mathbf{B}
\end{array}\right]\left[\begin{array}{l}
\mathbf{U} \\
\mathbf{V}
\end{array}\right]=k_{z}\left[\begin{array}{l}
\mathbf{U} \\
\mathbf{V}
\end{array}\right]
$$

By applying the surface wave conditions in which the surface waves propagate in a half space $(z>0)$, only $3 n$ eigenvalues in (6), which attenuate in the positive $z$ direction are chosen, i.e., $\operatorname{Im}\left(k_{z}\right)>0$. Then the tractionfree boundary conditions, $\left.\left.\mathbf{T}_{i 3}\right|_{z=0} \equiv C_{i 3 m n} \partial_{n} \mathbf{u}_{m}\right|_{z=0}=0$, on the surface $z=0$ are used in the calculations. T denotes the traction and $i=1,2,3$. Therefore, the dispersion relation for the surface waves propagating in the twodimensional phononic crystals, with both of the filling and background materials belonging to the triclinic system, can be obtained [17]. When $k_{z}$ in (5) is equal to zero, the equation degenerates into the eigenvalue problem of the bulk waves as:

$$
\mathbf{C} \cdot \mathbf{U}=0
$$

The dispersion relations of the bulk waves propagating in the two-dimensional phononic crystals can be obtained by setting the determinant of matrix $\mathbf{C}$ equal to zero. For materials with a symmetry higher than the orthorhombic symmetry, the matrix $\mathbf{C}$ can be decoupled into two different polarization modes. They are mixed polarization modes ( $\mathrm{SH}$ and $\mathrm{L}$, shear horizontal and longitudinal modes with polarization lies in the plane perpendicular to the fillers) and transverse polarization modes (SV, shear vertical mode with polarization along the filler direction). As the elastic waves propagate along the $x$ axis $(\Gamma-\mathrm{X}$ section), the nonvanishing displacements of the shear horizontal mode, shear vertical mode, and longitudinal mode are $u_{y}, u_{z}$, and $u_{x}$, respectively.

However, for materials with a symmetry lower than orthorhombic symmetry, the matrix $\mathbf{C}$ cannot be decoupled into two different polarization modes. The full matrix $\mathbf{C}$ must be considered and distinguished as quasi-SV, quasi$\mathrm{SH}$, and quasi-L modes.

\section{Temperature Effect on the Bandgaps of BAW and SAW in Phononic Crystals}

Consider phononic structures consisting of circular cylinders and a background material forming a twodimensional square lattice with lattice spacing $a(10 \mu \mathrm{m}$ for air/quartz). Air and quartz are used as the cylinders and the background materials, respectively. From the elastic constants and density at room temperature $\left(25^{\circ} \mathrm{C}\right)$, the temperature-dependent elastic constants and densities of the material, quartz, can be obtained by using [21]:

$$
\begin{aligned}
X(T) & \approx X\left(T_{0}\right)\left[1+\frac{1}{X\left(T_{0}\right)} \frac{\partial X}{\partial T}\left(T-T_{0}\right)\right. \\
& \left.+\frac{1}{2 X\left(T_{0}\right)} \frac{\partial^{2} X}{\partial T^{2}}\left(T-T_{0}\right)^{2}+\cdots\right],
\end{aligned}
$$

where $T_{0}$ is $25^{\circ} \mathrm{C}$, and $X\left(T_{0}\right)$ is the elastic constant evaluated at room temperature. $\frac{1}{X\left(T_{0}\right)} \frac{\partial X}{\partial T}$ and $\frac{1}{2 X\left(T_{0}\right)} \frac{\partial^{2} X}{\partial T^{2}}$ are the first and the second order temperature coefficients, respectively. $T$ is increased from 0 to $50^{\circ} \mathrm{C}$. The first order temperature coefficient of the density reads:

$$
\frac{1}{\rho\left(T_{0}\right)} \frac{\partial \rho}{\partial T}=-\left(\alpha_{11}+\alpha_{22}+\alpha_{33}\right)
$$

where $\alpha_{11}, \alpha_{22}$, and $\alpha_{33}$ are the expansion coefficients along the $x, y$, and $z$ axis, respectively.

In this paper, we only considered the first order temperature coefficients and expansion coefficients of quartz (Z-cut). The related constants can be found in [22] and the thermodynamic properties (density and sound speed) of air can be found in [23]. It is worth noting that the filling fraction and the effects of the thermal stresses arising from the thermal expansion mismatch between the superlattice components when the temperature is varied are neglected due to the air/solid band structure. 


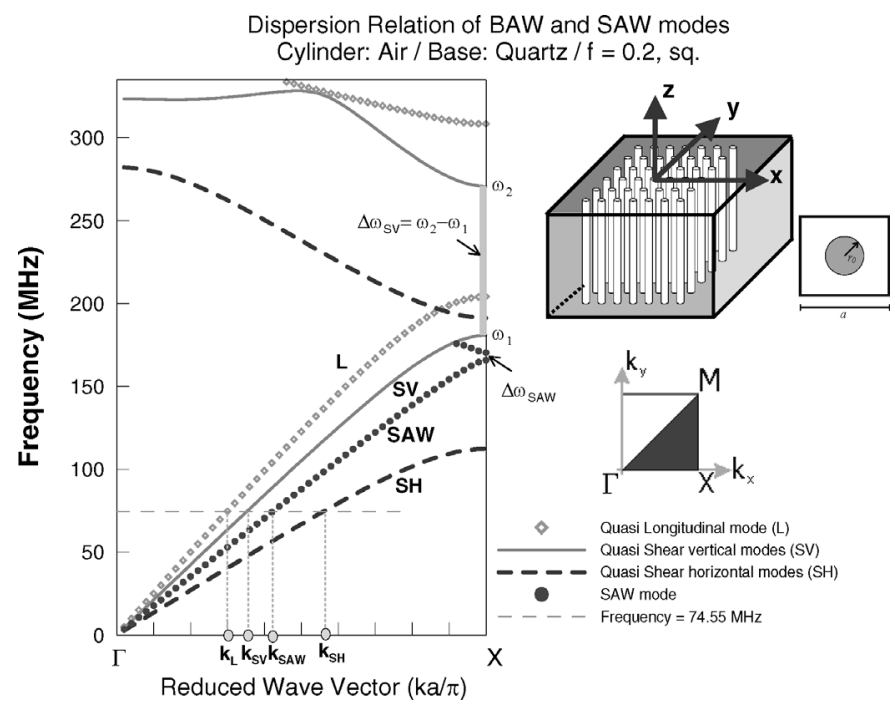

Fig. 1. Dispersion relations of the quasi-SV, quasi-SH, quasi-L, and SAW modes in air/quartz phononic structure with square lattice. The bandgap width is defined as $\Delta \omega=\omega_{2}-\omega_{1}$, and the lattice constant is $10 \mu \mathrm{m}$. The upper right diagram shows the system of circular rods (air) embedded in a background material (quartz) with square lattice. The lower right diagram shows the Brillouin zone of the square lattice. The shadow in the Brillouin zone is the irreducible part of the Brillouin zone. The filling fraction is defined as $f=$ $\left(\pi r_{0}^{2}\right) / a^{2}$.

The case considered in this paper is that we increase the temperature of the air/quartz band structure from 0 to $50^{\circ} \mathrm{C}$. The phononic crystal consists of quartz substrate and square arrays of air cylindrical holes. The modified PWE method can be applied because of the high-density contrast between solid and air [7], [8]. It is well-known that the high density contrast between solid and air leads to unexpected flat bands in the band structure. We get around this problem by adopting the technique shown in [7]. The flat bands can be removed by taking an artificial transverse velocity inside the fluid. Instead of postulating a purely longitudinal behavior of the solid [8], we also give an artificial transverse character to the fluid [7].

Fig. 1 shows the dispersion relations of the surface and bulk modes along the $\Gamma$-X section in the irreducible part of the Brillouin zone (see insert of Fig. 1) in air/quartz band structure with a filling ratio of 0.2 . In Fig. 1, the upper right diagram shows the system of circular rods (air) embedded in a background material (quartz) with square lattice; the lower right diagram shows the Brillouin zone of the square lattice. In the calculations, the $x-y$ plane is parallel to the (001) plane and the $x$ axis is along the [100] direction of quartz; the elastic properties and densities of the materials from 0 to $50^{\circ} \mathrm{C}$ used in this example are calculated from (8) and (9). The vertical axis is the frequency in megahertz unit, and the horizontal axis is the reduced wave vector $k^{*}=k a / \pi$. $k$ is the wave vector along the Brillouin zone. The thin solid lines represent the fundamental and higher quasi-SV modes, and the diamond symbols represent the quasi-L modes. The thin dashed lines represent the quasi-SH modes, and solid cir-

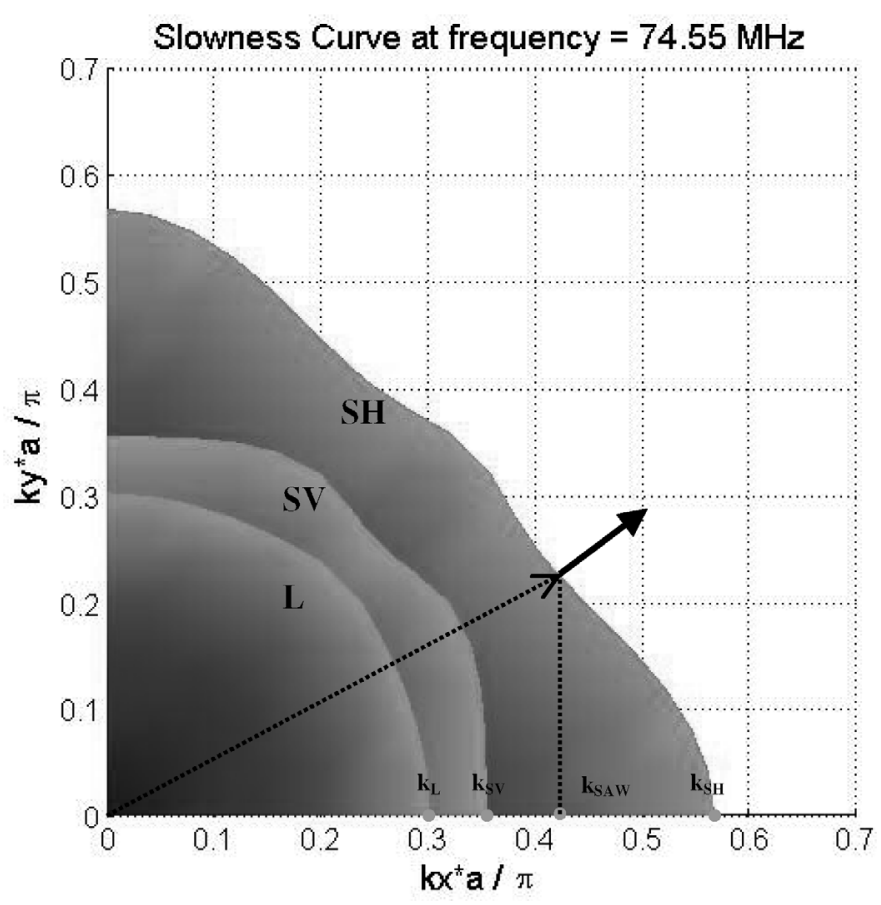

Fig. 2. The slowness curves of the bulk waves at the constant frequency $74.55 \mathrm{MHz}$ in the $k_{x}-k_{y}$ plane in a two-dimensional view.

cles represent the surface acoustic modes. The bandgap width is defined as $\Delta \omega=\omega_{2}-\omega_{1}\left(\omega_{1}, \omega_{2}\right.$ are the frequencies at the $\mathrm{X}$ point) and $\Delta \omega_{S V}, \Delta \omega_{S H}, \Delta \omega_{L}$, and $\Delta \omega_{S A W}$ represent the bandgap widths for quasi-SV, quasi$\mathrm{SH}$, quasi-L, and SAW modes, respectively. At room temperature, the bandgap widths for the above four types of modes are $\Delta \omega_{S V}=89.98 \mathrm{MHz}, \Delta \omega_{S H}=78.04 \mathrm{MHz}$, $\Delta \omega_{L}=104.76 \mathrm{MHz}$, and $\Delta \omega_{S A W}=4.77 \mathrm{MHz}$.

To understand the phenomena of bulk modes in the dispersion curves, the slowness curves of the three bulk modes in the $k_{x}-k_{y}$ plane are shown in Fig. 2. The slowness curves are plotted at the constant frequency $74.55 \mathrm{~Hz}$ in a two-dimensional view. It is worth noting that the curves of the three bulk modes are all deformed considerably from a circle due to both the material as well as geometrical anisotropy. However, the displacement fields of the surface modes are illustrative to see the magnitudes of the displacement components computed at the edge frequencies of the bandgap both in the $x-y$ plane and as a function of distance from the surface at some selected point. Shown in Fig. 3 are the relative amplitudes of the displacement components computed at frequency $\omega=\omega_{1}$ at $\mathrm{X}$ point. The selected point is the center of the cylinder. We found that the relative amplitudes of the displacement components on the surface consisted of three components, but the wave is mainly polarized as longitudinal. The inset in Fig. 3 is the magnitudes of the displacement components in the $x$ $y$ plane of a unit cell. The relative amplitudes in the inset are equal to $\left|u_{x}\right| /\left(u_{x}^{2}+u_{z}^{2}\right)^{1 / 2}$, in which we neglect the effect of component $u_{y}$. Similarly, in Fig. 4 are the relative amplitudes of the displacement components computed at frequency $\omega=\omega_{2}$ at $\mathrm{X}$ point. The displacement compo- 


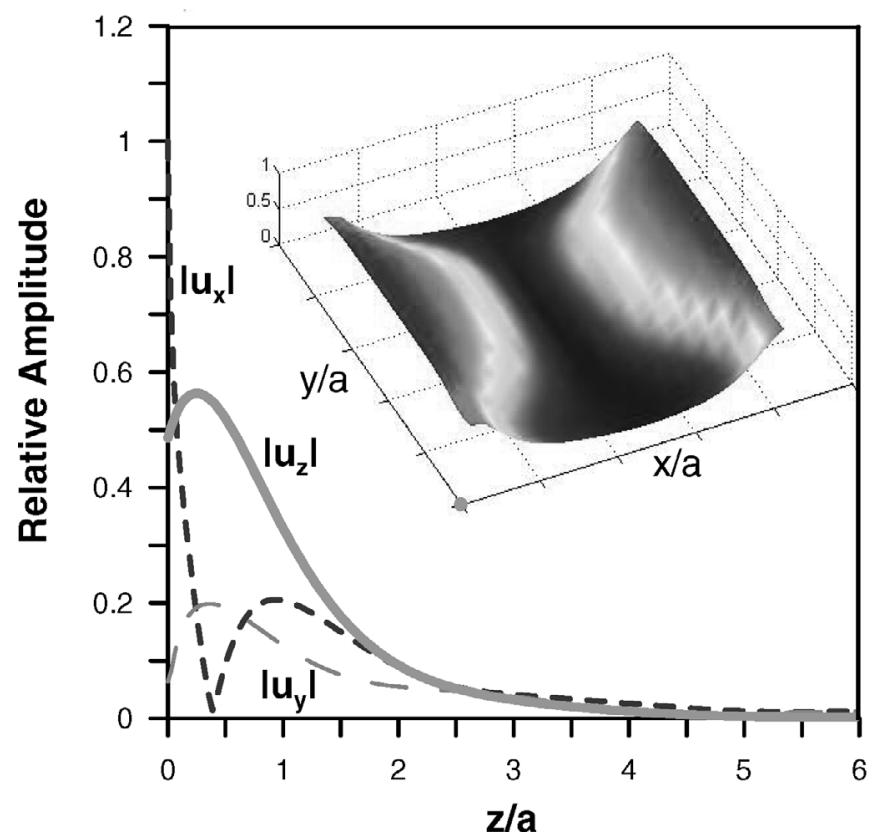

Fig. 3. Relative amplitudes of the displacement components of surface modes computed at frequency $\omega=\omega_{1}$ at X point. The selected point is the center of the cylinder. The inset is the magnitudes of the displacement components in the $x-y$ plane of a unit cell. The relative amplitudes in the inset are equal to $\left|u_{x}\right| /\left(u_{x}^{2}+u_{z}^{2}\right)^{1 / 2}$.

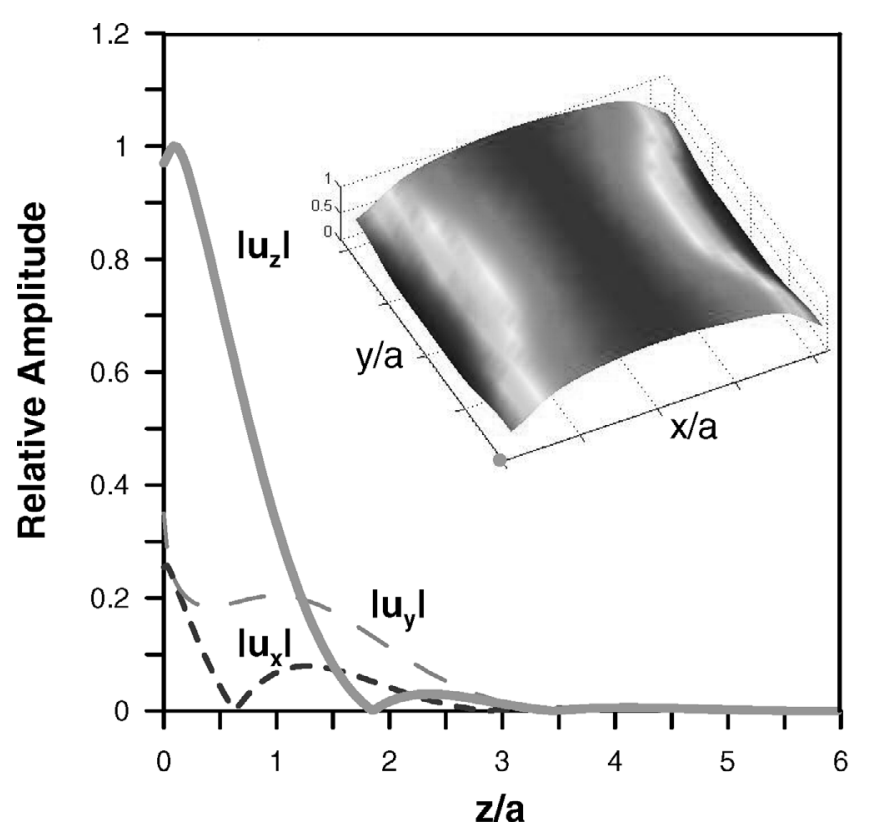

Fig. 4. Relative amplitudes of the displacement components of surface modes computed at frequency $\omega=\omega_{2}$ at X point. The selected point is the center of the cylinder. The inset is the magnitudes of the displacement components in the $\mathrm{x}-\mathrm{y}$ plane of a unit cell. The definition of the relative amplitudes is the same as Fig. 3.

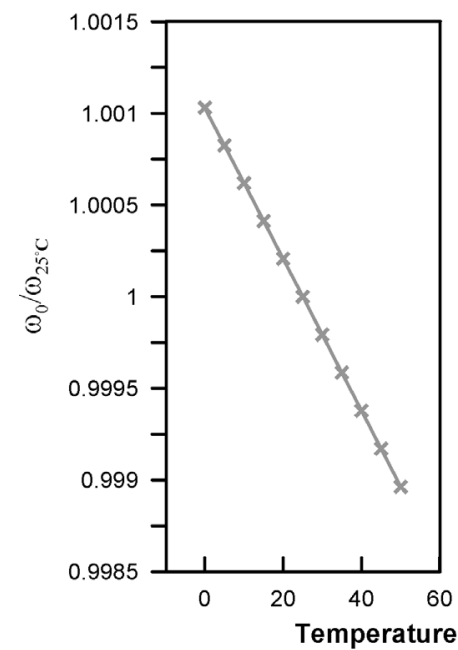

(a)

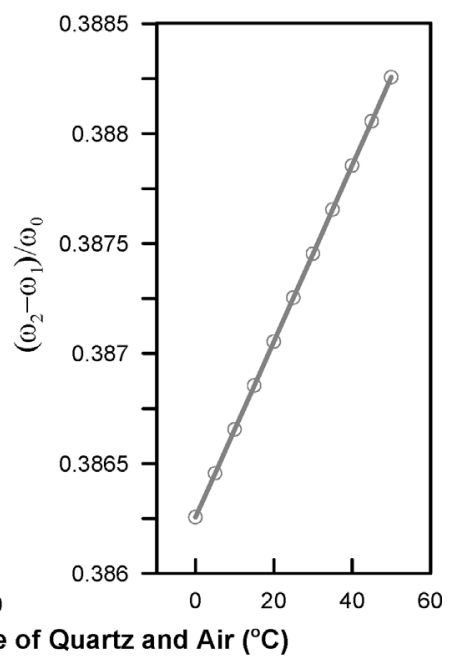

(b)
Fig. 5. Normalized center frequency of the gap and relative bandgap width of quasi-SV modes at X point.

nents on the surface consist of three components, but the wave is mainly polarized perpendicular to the surface. The inset in Fig. 4 is also the magnitudes of the displacement components in the $x-y$ plane of a unit cell. It is worth noting that the velocity of surface modes propagating along the $x$ axis in this air/quartz band structure is higher than that of the SH wave. The behavior is different from that in the case of a homogeneous material, in which the surface wave velocity is always slower than that of the shear wave.

With the temperature of the band structure being increased from 0 to $50^{\circ} \mathrm{C}$, Fig. 5 shows the center frequency of the gap [Fig. 5(a)] and relative bandgap width [Fig. 5(b)] of quasi-SV modes at $\mathrm{X}$ point. The center frequency $\omega_{0}$ is calculated as a function of temperature, normalized to the center frequency at $25^{\circ} \mathrm{C}$. Alternatively, the bandgap is plotted as relative bandgap width $\left(\omega_{2}-\omega_{1}\right) / \omega_{0}$ to allow better judgment of the temperature dependence. The horizontal axis of Fig. 5 is the temperature which varies from 0 to $50^{\circ} \mathrm{C}$. For the quasi-SV modes, we found that the frequencies $\omega_{1}$ and $\omega_{2}$ at point $\mathrm{X}$ decrease as the temperature rises. The normalized center frequency has the same phenomenon and the relative bandgap width increases as the temperature rises. Similarly, Figs. $6-8$ show the normalized center frequencies of the gap and relative bandgap widths of the quasi-SH, quasi-L, and SAW modes at $\mathrm{X}$ point, respectively. The results in quasi-SH modes show that the $\omega_{1}$ and $\omega_{2}$ at point $X$ increase as the temperature rises. The normalized center frequency and the relative bandgap width of the quasi-SH modes increase as the temperature rises shown in Fig. 6. At point $\mathrm{X}$, the $\omega_{1}$ of the quasi-L modes decreases, and $\omega_{2}$ increases as the temperature rises. This brings about that the normalized center frequency of the quasi-L modes remains constant with $10^{-5}$ accuracy [Fig. 7(a)]. However, the relative bandgap width increases as the temperature rises [Fig. 7(b)]. In regard of the SAW 
Center frequency of the gap and relative bandgap width of quasi-SH modes at $X$ point

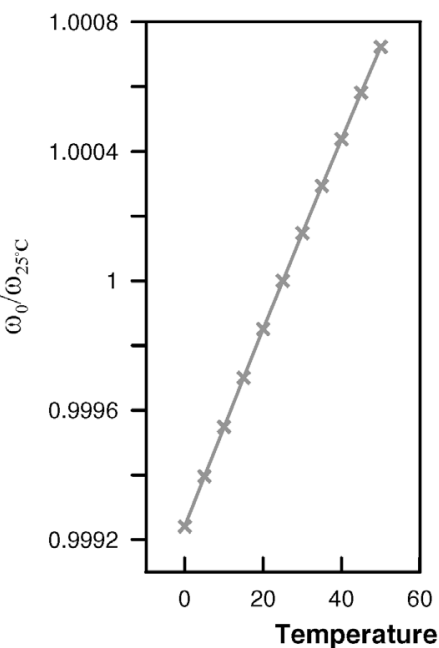

(a)

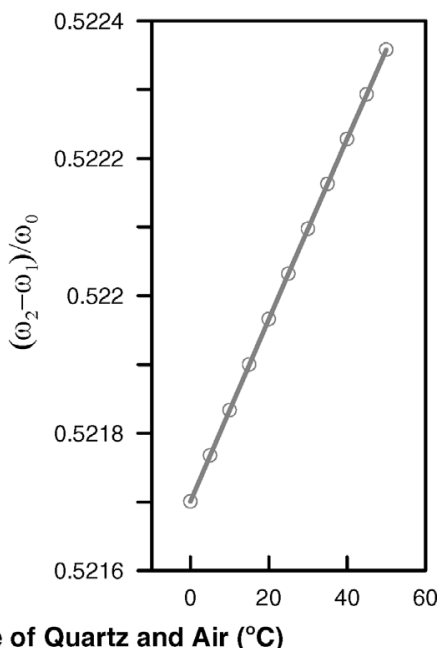

(b)
Fig. 6. Normalized center frequency of the gap and relative bandgap width of quasi-SH modes at $\mathrm{X}$ point.

Center frequency of the gap and relative bandgap width of quasi- $\mathrm{L}$ modes at $\mathrm{X}$ point

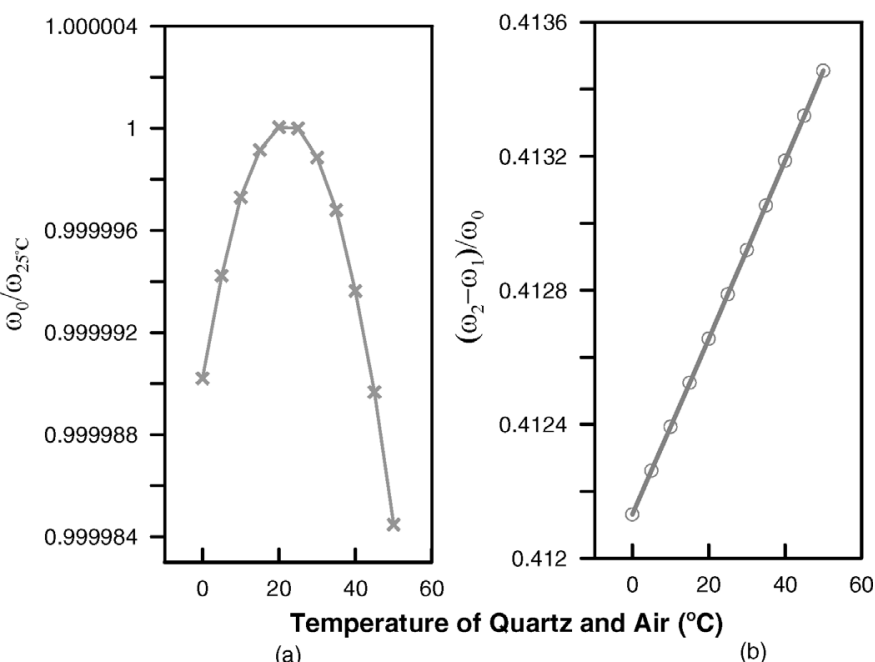

(a)

(b)

Fig. 7. Normalized center frequency of the gap and relative bandgap width of quasi-L modes at $\mathrm{X}$ point.

modes, the $\omega_{1}$ and $\omega_{2}$ frequencies at point $\mathrm{X}$ decrease as the temperature rises. The normalized center frequency also decreases, and the relative bandgap width increases as the temperature rises. It is worth noting that the relative bandgap widths of quasi-SV, quasi-SH, quasi-L, and SAW modes in Figs. 5-8 all increase in direct proportion to the temperature change.

\section{Summary and Conclusions}

Some important conclusions can be drawn from the above studies. First, we found that the temperature effects are obvious for all types of modes in the air/quartz band
Center frequency of the gap and relative bandgap width of SAW modes at $X$ point

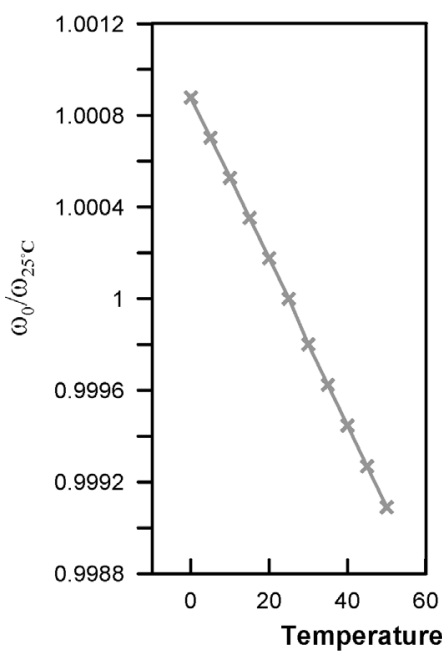

(a)

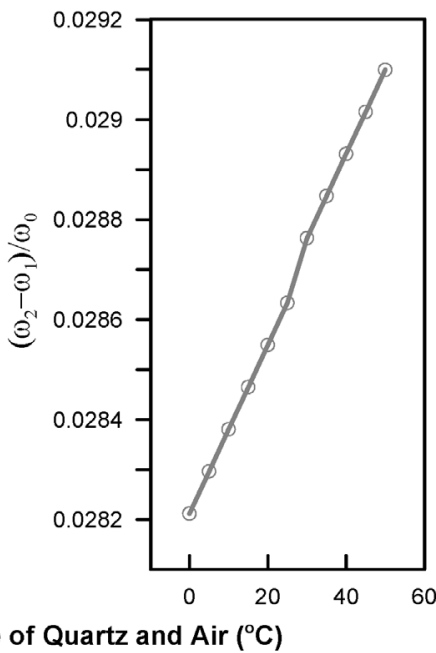

(b)
Fig. 8. Normalized center frequency of the gap and relative bandgap width of SAW modes at X point.

structure. Second, the tendencies of the relative bandgap widths for the four type modes are the same in air/quartz band structure, and the variations of the frequencies at $\mathrm{X}$ point are quite different. The frequencies $\omega_{1}$ and $\omega_{2}$ would decrease as the temperature rises. However, the $\omega_{1}$ and $\omega_{2}$ of quasi-SH modes and the $\omega_{2}$ of quasi-L modes in the air/quartz band structure increase as the temperature rises. We note that the bandgap width can be enlarged or reduced by adjusting the temperature of the band structure. These prominent features of the temperature effect on the bandgap variations of the phononic crystals may have potential applications in fine-tuning of the phononic bandgap, and they are very important on the precise filter design.

\section{ACKNOWLEDGMENT}

The authors gratefully acknowledge the National Science Council and the NTU-ITRI center of Taiwan for the financial support.

\section{REFERENCES}

[1] M. S. Kushwaha, P. Halevi, L. Dobrzynski, and B. DjafariRouhani, "Acoustic band structure of periodic elastic composites," Phys. Rev. Lett., vol. 71, no. 13, pp. 2022-2025, 1993.

[2] M. S. Kushwaha and P. Halevi, "Band-gap engineering in periodic elastic composites," Appl. Phys. Lett., vol. 64, no. 9, pp. 1085-1087, 1994.

[3] M. S. Kushwaha, P. Halevi, G. Martinez, L. Dobrzynski, and B. Djafari-Rouhani, "Theory of acoustic band structure of periodic elastic composites," Phys. Rev. B, vol. 49, no. 4, pp. 2313-2322, 1994.

[4] J. O. Vasseur, B. Djafari-Rouhani, L. Dobrzynski, M. S. Kushwaha, and P. Halevi, "Complete acoustic band gaps in periodic fibre reinforced composite materials: The carbon/epoxy composite and some metallic systems," J. Phys.: Condens. Matter, vol. 6, pp. 8759-8770, 1994. 
[5] J. O. Vasseur, P. A. Deymier, G. Frantziskonis, G. Hong, B. Djafari-Rouhani, and L. Dobrzynski, "Experimental evidence for the existence of absolute acoustic band gaps in two-dimensional periodic composite media," J. Phys.: Condens. Matter, vol. 10, pp. 6051-6064, 1998.

[6] M. Wilm, A. Khelif, S. Ballandras, and V. Laude, "Out-of-plane propagation of elastic waves in two-dimensional phononic bandgap materials," Phys. Rev. E, vol. 67, no. 6, article no. 065602, 2003.

[7] C. Goffaux and J. P. Vigneron, "Theoretical study of a tunable phononic band gap system," Phys. Rev. B, vol. 64, no. 7, article no. $075118,2001$.

[8] X. Li, F. Wu, H. Hu, S. Zhong, and Y. Liu, "Large acoustic band gaps created by rotating square rods in two-dimensional periodic composites," J. Phys. D: Appl. Phys., vol. 36, pp. L15L17, 2003.

[9] M. Kafesaki and E. N. Economou, "Multiple-scattering theory for three-dimensional periodic acoustic composites," Phys. Rev. B, vol. 60, no. 17, pp. 11993-12001, 1999.

[10] I. E. Psarobas and N. Stefanou, "Scattering of elastic waves by periodic arrays of spherical bodies," Phys. Rev. B, vol. 62, no. 1, pp. 278-291, 2000.

[11] Z. Liu, C. T. Chan, and P. Sheng, "Elastic wave scattering by periodic structures of spherical objects: Theory and experiment," Phys. Rev. B, vol. 62, no. 4, pp. 2446-2457, 2000.

[12] J. Mei, Z. Liu, J. Shi, and D. Tian, "Theory for elastic wave scattering by a two-dimensional periodical array of cylinders: An ideal approach for band-structure calculations," Phys. Rev. B, vol. 67, no. 24, article no. 245107, 2003.

[13] D. Garcia-Pablos, M. Sigalas, F. R. Montero de Espinosa, M. Kafesaki, and N. Garcia, "Theory and experiments on elastic band gaps," Phys. Rev. Lett., vol. 84, no. 19, pp. 4349-4352, 2000 .

[14] T. Aono and S. Tamura, "Surface and pseudosurface acoustic waves in superlattices," Phys. Rev. B, vol. 58, no. 8, pp. 48384845, 1998.

[15] Y. Tanaka and S. Tamura, "Surface acoustic waves in twodimensional periodic elastic structures," Phys. Rev. B, vol. 58, no. 12 , pp. $7958-7965,1998$.

[16] _ , "Acoustic stop bands of surface and bulk modes in twodimensional phononic lattices consisting of aluminum and a polymer," Phys. Rev. B, vol. 60, no. 19, pp. 13294-13297, 1999.

[17] T.-T. Wu, Z.-G. Huang, and S. Lin, "Surface and bulk acoustic waves in two-dimensional phononic crystals consisting of materials with general anisotropy," Phys. Rev. B, vol. 69, no. 9, article no. 094301, 2004.

[18] R. E. Vines, J. P. Wolfe, and A. G. Every, "Scanning phononic lattices with ultrasound," Phys. Rev. B, vol. 60, no. 17, pp. 11871-11874, 1999.
[19] R. E. Vines and J. P. Wolfe, "Scanning phononic lattices with surface acoustic waves," Physica B, vol. 263-264, pp. 567-570, 1999.

[20] A. G. Every, R. E. Vines, and J. P. Wolfe, "Line-focus probe excitation of Scholte acoustic waves at the liquid-loaded surfaces of periodic structures," Phys. Rev. B, vol. 60, no. 16, pp. 1175511760, 1999.

[21] A. A. Oliner, Acoustic Surface Waves-Topics in Applied Physics 24. Berlin: Springer-Verlag, 1978.

[22] M. B. Schulz, B. J. Matsinger, and M. G. Holland, "Temperature dependence of surface acoustic wave velocity on $\alpha$ quartz," $J$. Appl. Phys., vol. 41, no. 7, pp. 2755-2765, 1970.

[23] D. R. Lide, CRC Handbook of Chemistry and Physics. 83rd ed. Boca Raton, FL: CRC Press, 2003.

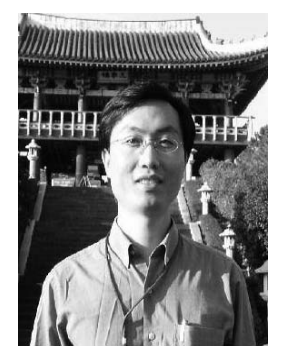

Zi-Gui Huang received the B.S. degree in the department of Aeronautics and Astronautics from National Cheng Kung University and M.S. degree in the Institute of Applied Mechanics from National Taiwan University in 1998 and 2000 respectively. Presently, he is a Ph.D. candidate in the Institute of Applied Mechanics, National Taiwan University. His research work is mainly on the Phononic Crystals and the Vibration of Piezoelectric Plates.

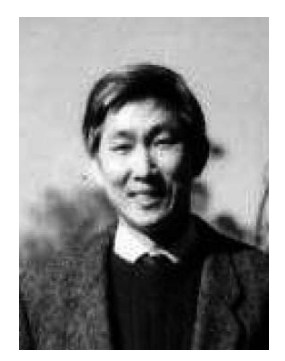

Tsung-Tsong $\mathbf{W u}$ received his doctorate in Theoretical and Applied Mechanics from Cornell University in 1987. Then, he joined the National Taiwan University faculty and is now a professor of the Institute of Applied Mechanics. He was awarded the distinguished research prizes of the National Science Council (NSC) three times for six years from 1995 to 2001 and is currently a distinguished research fellow of NSC. He is on the international advisory board of the journal NDT\&E International, the executive board director of the Taiwanese Society of Nondestructive Testing, and the executive board director of the Quartz Industry Association of Taiwan. He is a member of the American Society of Mechanical Engineers and American Society for Nondestructive Testing. 